\title{
EDUCAÇÃO PREVENTIVA AO ABUSO DE DROGAS EM ESCOLAS PÚBLICAS NUM MUNICÍPIO DO SUL DO BRASIL.
}

\author{
M. F. KNEVITZ * , J. U. BÉRIA, L. B. SHERMANN \\ Universidade Luterana do Brasil \\ marcosknevitz@hotmail.com*
}

Submetido 30/07/2016 - Aceito 07/06/2018

DOI: $10.15628 /$ holos.2018.4896

\section{RESUMO}

A presente pesquisa objetivou conhecer práticas preventivas ao abuso de drogas em escolas públicas municipais e estaduais. Tratou-se de um estudo descritivo, para a realização do qual foram utilizados dois questionários semiestruturados, respondidos pelas direções e professores. Os resultados encontrados apontaram que o tema drogas é trabalhado em $87,5 \%$ das escolas, estando registrado no projeto pedagógico em $57,5 \%$ dessas escolas. As atividades de educação preventiva com maior frequência foram palestras e distribuição de material educativo, sendo que $24 \%$ dos professores se consideraram preparados para atuar na educação preventiva. Os profissionais da educação demonstraram necessidade de orientação sobre como trabalhar com o tema. A prevenção pode ser melhorada com uma maior interação entre profissionais técnicos e a comunidade escolar, além de garantia de formação adequada aos professores.

PALAVRAS-CHAVE: Instituições acadêmicas, Docentes, Transtornos relacionados ao uso de substâncias.

\section{PREVENTIVE EDUCATION TO DRUGS ABUSE IN PUBLIC SCHOOLS ON A SOUTHERN BRAZIL CITY.}

\begin{abstract}
The present study aimed to know preventive practices to drug abuse in municipal and state public schools. It was a descriptive study, for which two semi-structured questionnaires, answered by the directions and teachers, were used. The results showed that the topic drugs is worked in $87.5 \%$ of schools, being registered in the educational project in $57.5 \%$ of these schools. The most frequent preventive education activities were
\end{abstract}

lectures and distribution of educational material, with $24 \%$ of teachers considered themselves prepared to act in preventive education. Education professionals have demonstrated a need for guidance on how to work with the topic. Prevention can be improved through increased interaction between technical professionals and the school community, as well as ensuring adequate training for teachers.

KEYWORDS: Schools, Faculty, Substance related disorders. 


\section{INTRODUÇÃO}

Estima-se que em 2014, um entre vinte adultos ou um quarto de bilhão de pessoas com idade entre 15 e 64 anos, correspondendo a entre 3,5\% e 7,0 \% da população mundial, tenha usado alguma droga ilícita, pelo menos uma vez no ano e apenas um em cada seis usuários tiveram acesso aos serviços de tratamento (UNODC, 2016). A consequência disso é o aumento na carga global de doença, anos de vida produtiva perdidos (Degenhardt et al, 2010) e uma taxa de mortalidade de cerca de 40 mortes por milhão (UNODC, 2016). Acabar com a possibilidade do uso de drogas entre os seres humanos é tarefa impraticável, pois seria necessário modificar a própria condição ontológica do homem. Sendo assim, deve ser prudente que as ações de promoção da saúde e prevenção ao uso abusivo de drogas fossem voltadas para que o próprio sujeito pudesse ter condições de refletir e estabelecer uma melhor relação com as substâncias psicoativas (SPAs) (Sodelli, 2010).

O aumento da prevalência no consumo abusivo de SPAs, a precocidade do seu uso e as dificuldades em restringir o acesso são inquietações no Brasil e a gestão pública está buscando um enfrentamento mais efetivo desse problema a partir da elaboração de preceitos que orientem os envolvidos com a questão (Horta et al, 2014). O marco legal deste momento de confronto com a drogadição foi a promulgação da lei 11343 de 23 de agosto de 2006 (Lei das Drogas), que institui o Sistema Nacional de Políticas Públicas sobre Drogas, que prescreve medidas para a prevenção do uso indevido, atenção e reinserção social de usuários e dependentes de drogas; estabelece normas para repressão à produção não autorizada e ao tráfico ilícito de drogas; define crimes e dá outras providências (Brasil, 2006).

A Lei das Drogas contempla os aspectos da educação no seu artigo 19, o qual dispõe sobre o estabelecimento de políticas de formação continuada na área da prevenção do uso indevido de drogas para os professores, nos três níveis de ensino. Além disso, refere a necessidade da implantação de projetos pedagógicos de prevenção nas instituições de ensino público e privado, alinhados às diretrizes curriculares nacionais e aos conhecimentos relacionados a drogas (Brasil, 2006).

As escolas têm sido apontadas como espaços privilegiados para o desenvolvimento de atividades preventivas e promotoras da saúde, pois a maioria da população frequenta esses estabelecimentos e pode proporcionar circunstâncias favoráveis para a assimilação de certos hábitos, atitudes e informações. Ou seja, além da sua função de ensino, as escolas constituem um espaço de socialização e construção da identidade fora da família, criando condições para a produção e acesso ao conhecimento socialmente produzido (Santos et al, 2011). Apesar dessas condições favoráveis, os estabelecimentos de ensino estão com dificuldades para atuar na educação preventiva ao abuso de álcool e outras drogas, pois os professores, apesar de reconhecerem seu papel de formadores de opinião, não se sentem motivados (Araldi et al, 2012) ou preparados (Knevitz, Béria e Schermann, 2017) para trabalhar com o tema.

No Brasil, as orientações que os estabelecimentos de ensino recebem do Ministério da Educação, com relação aos temas da saúde, estão nos Parâmetros Curriculares Nacionais (PCNs), 
que estabelecem as diretrizes elaboradas pelo governo federal e orientam a educação. As situações geradas pelo uso de SPAs devem ser abordadas no tema vida coletiva e serem tratadas em todas as áreas que compõem o currículo escolar, de forma multidisciplinar, pois fazem parte dos chamados temas transversais (Brasil, 2013a).

Para integrar as políticas de saúde com as da educação foi criado o Programa Saúde na Escola (PSE), o qual visa contribuir para a formação integral dos estudantes de escolas públicas com ações relativas à saúde. Pelas bases do PSE, os profissionais das Equipes de Saúde da Família (ESF), estabelecem contato direto com os estabelecimentos de ensino para realizar a avaliação das condições de saúde dos estudantes e realizar intervenções, abrangendo a prevenção ao uso abusivo de SPAs (Brasil, 2007). Embora seja uma de suas atribuições mais importantes, os profissionais do PSE abordam pouco os temas relacionados ao abuso de SPAs. Talvez isso ocorra em função da falta de subsídios para ações voltadas à prevenção ou por carecerem de informações técnicas gerais que envolvam o assunto (Barros, 2007).

Considera-se a dimensão que o ambiente escolar ocupa na socialização dos jovens, podendo proporcionar condições para a promoção à saúde e prevenção ao uso abusivo de álcool e outras drogas, contudo para que os estabelecimentos de ensino pudessem exercer esse potencial deveriam estar capacitados tecnicamente. Sendo assim, o presente estudo tem por objetivo conhecer as práticas preventivas ao abuso de álcool e outras drogas nas escolas públicas de ensino fundamental e médio de uma cidade da região metropolitana de Porto Alegre, além de verificar a experiência dos educadores e suas demandas quanto ao tema.

\section{METODOLOGIA}

Foi realizado um estudo descritivo com as equipes diretivas e com todos os professores de ensino fundamental e médio das escolas públicas municipais e estaduais do município de Santo Antônio da Patrulha, RS. O projeto desta pesquisa foi aprovado pelo Comitê de Ética da ULBRA, através da Plataforma Brasil, conforme o parecer no 702.998 de 24 de junho de 2014 e CAE no 32093114.8.0000.5349.

Previamente a coleta de dados, foi realizado um estudo piloto, com duas escolas públicas municipais em um município vizinho, para adequação tanto dos instrumentos quanto do procedimento de coleta de dados. A pesquisa foi desenvolvida com as equipes diretivas das 40 escolas públicas do município, sendo 16 municipais e 24 estaduais, com a população de professores registrados, que somava 473 professores estaduais registrados pela 11 a $\mathrm{CRE}$ (Coordenadoria Regional de Educação) e 243 professores municipais registrados pela SEMED (Secretaria Municipal de Educação), totalizando 716 professores. No entanto, foi constatado que um mesmo professor poderia estar registrado em mais de uma matrícula e/ou mais de um estabelecimento. Assim, foi realizada nova verificação, desconsiderando matriculas e/ou funções duplicadas e profissionais fora da escola. $O$ total de professores aptos a responder o questionário ficou em 162 municipais e 336 estaduais, dos quais, respectivamente, seis e 42 professores se recusaram ou não devolveram o questionário. A população final totalizou 450 professores, 156 municipais e 294 estaduais. 
Foram utilizados, como instrumentos, dois questionários semiestruturados. O primeiro foi respondido pelas equipes diretivas das escolas e buscou dados sobre a estrutura dos estabelecimentos e suas atitudes práticas sobre educação preventiva ao abuso de álcool e outras drogas. Foi baseado em instrumento utilizado pelo Ministério da Educação no censo escolar (Educacenso) e elaborado pelo Instituto Nacional de Estudos e Pesquisas Educacionais Anísio Teixeira (INEP) (Brasil, 2013b). O segundo questionário foi respondido pelos professores e coletou dados pessoais e profissionais, experiência e conhecimento sobre o assunto e as principais demandas acerca da questão da educação preventiva. Também foi baseado em instrumento utilizado pelo Ministério da Educação no censo escolar e preparado pelo INEP (Brasil, 2013b).

Para as escolas municipais, a coleta de dados foi realizada mediante a entrega dos questionários e do Termo de Consentimento Livre e Esclarecido (TCLE) para os diretores durante reunião mensal, que ocorreu na sede da SEMED. Nesta reunião, o pesquisador apresentou o estudo e explicou como seria a coleta de dados, o preenchimento dos questionários e a data de entrega dos mesmos. Os instrumentos foram recolhidos após duas semanas da entrega, na própria escola, por integrantes da SEMED ou pelo pesquisador. Com relação às escolas estaduais, a entrega dos questionários e do TCLE, bem como as orientações para o preenchimento foram realizadas nas próprias escolas com a presença do pesquisador. O recolhimento dos questionários foi feito da mesma maneira que nas escolas municipais.

Os dados coletados foram digitados no programa Epidata, com dupla digitação, para o controle de qualidade, onde foram compilados, organizados e transferidos para o pacote estatístico SPSS (Statistical Package for Social Sciences) versão 18.0 for Windows. Foi realizada análise descritiva e se avaliou possíveis associações, pelo teste qui-quadrado de Pearson. O nível de significância considerado na análise foi de $p<0,05$.

\section{RESULTADOS}

\subsection{Aspectos das escolas}

Dentre as 40 escolas estudadas, $60 \%$ eram estaduais e $40 \%$ municipais sendo que $72,5 \%$ estavam localizadas na zona rural do município. Tanto as escolas estaduais quanto as municipais desenvolviam, na mesma proporção, atividades sobre o tema álcool e outras drogas (87,5\%). A localização também mostrou resultados muito próximos, onde as escolas urbanas tinham 90,9\% e as rurais $86,2 \%$ de estabelecimentos que atuam na questão.

O tema álcool e outras drogas estava incluído no projeto pedagógico em $57,5 \%$ das escolas. Quando isto ocorre o assunto sempre é trabalhado em sala de aula. Das escolas que não possuem o tema incluído, $70,6 \%$ trabalhavam com o mesmo, sendo esta diferença significativa $(p=0,005)$.

As atividades desenvolvidas em educação preventiva (EP) que tiveram a maior frequência foram palestras em $55 \%$ dos estabelecimentos e distribuição de material educativo em $35 \%$. Projetos específicos sobre drogas, que são atividades que propõem uma maior abrangência de ações, foram desenvolvidos em apenas $12,5 \%$ das escolas. 
Os profissionais responsáveis pelas atividades eram principalmente professores não capacitados em EP em 57,5\% dos estabelecimentos e profissionais de saúde de nível superior em $30 \%$. A frequência dessas atividades ocorreram anualmente em $35 \%$ e semestralmente em $22,5 \%$ dos casos.

O PSE esteve presente em $37,5 \%$ das escolas do município, oferecendo atividades preventivas em $25 \%$ delas. As atividades realizadas foram as palestras em $100 \%$ dos casos, distribuição de material educativo em $40 \%$, enquanto que a realização de projetos específicos sobre drogas ocorreu em apenas $10 \%$ dos trabalhos. Os principais motivos da escola não contar com o PSE foram ele não ter sido oferecido (40\%) e faltar conhecimento sobre o programa $(22,5 \%)$.

\subsection{Aspectos dos professores}

Dos 450 professores que participaram da pesquisa, $92 \%$ eram do sexo feminino, $56 \%$ com idade entre 30 e 49 anos, 56,2\% eram casados e 67,3\% tinham filhos. Quanto à escolaridade, $88,4 \%$ tinha curso superior e $58,7 \%$ obtiveram esse nível nos últimos sete anos. Realizaram o curso superior de modo presencial $76,7 \%$. Possuíam alguma pós-graduação $63,3 \%$, sendo $55,6 \%$ em nível de especialização, 7,1\% atualização e 0,7\% mestrado. Estavam lecionando há mais de 16 anos, 45,6\% dos docentes e trabalhavam na mesma escola, há menos de 5 anos, 57,8\%.

Para chegar aos estabelecimentos de ensino, 67,1\% utilizavam meios próprios e 78,7\% levavam até meia hora para concluir o percurso. Trabalhavam em apenas uma escola 55,3\% e $86,9 \%$ dos professores não possuíam atividades laborais diferentes da educação. Com relação a educação preventiva, $97,8 \%$ dos entrevistados acreditavam que a escola deveria desenvolver atividades preventivas. Pouco mais da metade $(52,2 \%)$ já participou de atividades de formação na área de educação ao abuso de álcool e outras drogas, 23,1\% empregavam quase sempre os conhecimentos adquiridos nessas formações, $22,2 \%$ os usavam eventualmente e $6,9 \%$ não ou quase nunca utilizavam. Os conhecimentos adquiridos nestas formações foram empregados em sala de aula através de atividades preventivas, estão demonstrados na Tabela 1.

Tabela 1: Atividades em educação preventiva ao uso abusivo de álcool e outras drogas desenvolvidas em escolas públicas de Santo Antônio da Patrulha, RS, 2014.

\begin{tabular}{lcc}
\hline Atividade & $\mathrm{n}^{*}$ & $\%$ \\
\hline Palestras & 88 & 26,5 \\
Distribuição de material educativo & 70 & 21,1 \\
Projeto específico sobre drogas & 48 & 14,5 \\
Feira de ciências & 29 & 8,8 \\
Conversa/Diálogo & 25 & 7,5 \\
Oficinas & 21 & 6,3 \\
Texto/Leitura & 10 & 3,0 \\
Debates & 9 & 2,7 \\
Vídeo/Filme & 9 & 2,7 \\
Outros** & 23 & 6,9 \\
\hline Total & 332 & 100 \\
\hline
\end{tabular}


*Total de respostas à questão de múltipla escolha.

**Trabalho escolar, teatro, pesquisa, reflexões, aula expositiva, conversa com os pais, troca de experiências, cartazes, questionários e atividades de formação.

Os professores responderam também sobre as principais dificuldades que a escola tem em implantar e manter a Educação Preventiva, sendo os resultados demonstrados na Tabela 2.

Tabela 2: Dificuldades enfrentadas para implantar e manter a educação preventiva ao uso abusivo de álcool e outras drogas em escolas públicas de Santo Antônio da Patrulha, RS, 2014.

\begin{tabular}{lcc}
\hline Dificuldade & $\mathrm{n}^{*}$ & $\%$ \\
\hline Falta de profissionais capacitados sobre o tema & 238 & 19,6 \\
Baixa participação da família & 193 & 15,9 \\
Falta de conhecimento técnico sobre o assunto & 188 & 15,4 \\
Falta de políticas de incentivo a Educação Preventiva & 180 & 14,8 \\
Excesso de atribuições aos professores & 154 & 12,7 \\
Baixa participação da comunidade & 85 & 7,0 \\
Falta de orientação dos órgãos governamentais & 71 & 5,8 \\
Receio de represálias por parte dos traficantes & 37 & 3,0 \\
Falta de interesse dos profissionais da educação & 36 & 2,9 \\
Infraestrutura inadequada do estabelecimento de ensino & 32 & 2,6 \\
Outros** & 3 & 0,3 \\
\hline Total & 1217 & 100 \\
\hline
\end{tabular}

*Total de respostas à questão de múltipla escolha.

**Corrupção, falta de autoridade do poder público e leis insuficientes ou dúbias.

No geral, $24 \%$ dos professores disseram que se sentiam preparados para realizar atividades preventivas ao abuso de álcool e outras drogas. Além disso, foi analisada a influência que as atividades de formação na área de educação preventiva tiveram sobre os professores, quanto ao fato de se sentirem preparados para realizarem atividades preventivas. Observou-se uma diferença significativa $(p=0,000)$, onde $35,5 \%$ dos que participaram das atividades se sentiram preparados, enquanto que somente $12,6 \%$ se consideraram aptos, quando não realizaram as atividades.

Outra questão que chamou atenção, foi a dos professores que possuíam atividades diferentes da educação, onde $41,4 \%$ se sentiram preparados para realizarem atividades preventivas, enquanto que esse percentual baixou para $22,1 \%$ no que se refere aos profissionais que trabalham apenas com a educação, sendo essa diferença significativa ( $p=0,002)$. 


\section{DISCUSSÕES}

\subsection{Aspectos das escolas}

O presente estudo demonstrou que pouco mais da metade das escolas $(57,5 \%)$, possuíam o tema álcool e outras drogas no seu projeto político pedagógico (PPP). Quando se comparou os estabelecimentos que possuíam o tema no PPP com aqueles que não tinham, verificou-se uma diferença significativa com relação a trabalhar o tema em sala de aula. Quando o tema não estava no PPP, quase um terço $(29,5 \%)$ das escolas não trabalhava com o assunto, enquanto todas as escolas que o tinham registrado, realizavam atividades em sala de aula. Isso demonstra a importância em seguir as determinações da Lei das Drogas (Brasil, 2006), que estabelece a implementação da prevenção do uso indevido de drogas no projeto pedagógico das instituições de ensino. O que se verificou nas escolas do município é que, mesmo após oito anos de sua publicação, a lei não está sendo totalmente aplicada, diminuindo o acesso dos estudantes à temática.

Com relação ao tipo de atividades desenvolvidas, observou-se que as que tiveram a maior frequência foram palestras e distribuição de material informativo, que são ações pontuais e pouco interativas. Os programas escolares que possuem maior propensão à efetividade, são os que possuem atividades mais interativas e conseguem trabalhar o desenvolvimento das habilidades sociais dos alunos (Midford et al, 2012). Verificou-se ainda que o pequeno número de projetos específicos sobre drogas (12,5\%) é um indicativo de que existem poucas estratégias sistematizadas de prevenção (Araldi et al, 2012). Além disso, os professores não capacitados em educação preventiva, sendo os principais responsáveis pelas atividades realizadas, e a frequência dessas ações acontecendo anual ou semestralmente podem indicar que os docentes precisam de uma formação mais específica sobre o tema e que o planejamento das ações necessite de maior atenção pelos gestores públicos.

Nesse sentido, o direcionamento das ações em educação preventiva deveria ser orientado pelo trabalho interativo dos profissionais da educação e saúde. Entretanto, o PSE, que seria o programa oficial que trabalharia com promoção da saúde e daria suporte técnico para as escolas, quanto ao tema (Brasil, 2007), ainda está restrito a poucas escolas e oferece atividades preventivas ao uso abusivo do álcool e outras drogas em apenas um quarto dos estabelecimentos. Outrossim, as atividades oferecidas em prevenção são sempre palestras e, em $40 \%$ dos casos, a distribuição de material informativo, indicando uma carência de projetos mais interativos com os alunos e a comunidade. Um estudo realizado no interior de São Paulo demonstrou que faltariam subsídios aos profissionais da saúde, para realizar ações de prevenção de maneira mais efetiva (Barros, 2007).

Diante das questões discutidas, é possível afirmar que o processo preventivo permanece ainda incipiente nas escolas estudadas, sendo as principais atividades realizadas pouco interativas e de frequência baixa. O principal programa oficial (PSE), que poderia oferecer 
alternativas em prevenção, tem atuado pouco sobre o tema, mostrando-se insuficiente. Os profissionais que trabalham nas escolas teriam maior chance de efetividade se trabalhassem com a promoção da saúde (Kornblit, 2010), que é um dos pilares da lei das drogas (Brasil, 2006).

\subsection{Aspectos dos professores}

Em conformidade com os resultados do presente estudo, embora a quase totalidade dos entrevistados $(97,8 \%)$ tenha referido que a escola devesse trabalhar com o tema, pouco mais da metade $(52,2 \%)$ participou de atividades de formação em educação preventiva ao abuso de álcool e outras drogas e apenas $23,1 \%$ faziam uso frequente dos conhecimentos adquiridos nessas formações em suas escolas. Isto pode ser um indicativo de que as formações existentes não estejam atingindo os docentes de maneira satisfatória e quando o fazem, não estão conseguindo subsidiá-los com conhecimento e motivação, de forma a modificar suas atitudes. Apesar de diversos estudos apontarem a importância da instituição escola no processo da educação preventiva (Santos et al, 2011), sendo o professor considerado agente fundamental deste processo (Norberg, Kezelman e Lim, 2013), os profissionais da educação, no entanto, admitem que têm dificuldades em trabalhar com o tema em sala de aula (Araldi et al, 2012), corroborando os achados da presente pesquisa, onde menos de um quarto dos entrevistados utilizou as informações recebidas em capacitações.

Tendo em vista que as principais atividades em educação preventiva desenvolvidas pelos professores, confirmando o referido pelos gestores das escolas, foram palestras e distribuição de material informativo, que embora tenham importância, configuraram-se como tarefas realizadas esporadicamente e com menor participação dos alunos. Ademais, palestras também aparecem como o evento mais desenvolvido em uma escola no interior da Bahia, onde a comunidade escolar constatou falta de preparo diante da realidade das drogas no contexto escolar (Santos et al, 2011). Sendo assim, as ações de prevenção ao uso abusivo de substâncias psicoativas (SPAs) deveriam estar integradas às atividades curriculares dos estabelecimentos de ensino, dentro de um cronograma que proporcione continuidade do trabalho e participação dos estudantes no processo (Brasil, 2006).

Embora os achados da nossa pesquisa possam demonstrar que existiam problemas com a formação dos professores, os docentes que realizaram alguma capacitação sobre o tema, disseram estar aptos a realizar atividades preventivas com uma frequência três vezes maior quando comparado com os que não tiveram capacitação. Considerando que $24 \%$ do total de professores se declararam aptos a realizar atividades preventivas, fica evidente a necessidade do desenvolvimento de estratégias sistematizadas na formação dos docentes, para uma melhor perspectiva de resultados na educação preventiva.

É preciso que os professores estejam preparados para se envolver nos processos de ensino sobre SPAs, mas o grau de instrução sobre o tema pode variar conforme a jurisdição. Em British Columbia, no Canadá, nenhuma das principais instituições de formação de professores da Universidade de British Columbia, Simon Fraser University e da Universidade de Victoria, inclui educação sobre drogas como um componente central no currículo, apesar do fato de que os professores podem ser incumbidos de trabalhar a educação sobre drogas, como parte dos seus 
deveres profissionais (Tupper, 2008). Na Austrália, depois de um período de uma melhor formação de professores para a educação sobre drogas em meados da década de 1990, o desenvolvimento profissional nesta área tem diminuído nos últimos anos (Midford et al, 2007).

Alguns países, como a Argentina (Camarotti, Kornblit e Di Leo, 2013), oferecem cursos de capacitação em prevenção ao uso abusivo de drogas, específicos para professores, que proporcionam uma formação voltada para a promoção de saúde na escola. Os programas de prevenção de base escolar com os resultados mais efetivos na redução da incidência do uso abusivo de drogas são aqueles que consideram fatores sociais e psicológicos como relevantes e visam o desenvolvimento de habilidades na tomada de decisões e a percepção do dano que o uso inadequado dessas substâncias pode causar (Stephens et al, 2009).

No Brasil, a legislação sobre o uso de drogas regulamenta a formação continuada de professores, nos três níveis de ensino, também tendo a promoção da saúde como foco central (Brasil, 2006). A principal atividade de formação oferecida aos professores brasileiros é o curso de prevenção do uso de drogas para educadores de escolas públicas, promovido pela Secretaria Nacional de Políticas sobre Drogas (SENAD) do Ministério da Justiça e pela Secretaria de Educação Básica do Ministério da Educação (MEC). Esse curso está na sexta edição e ofereceu, em 2014, 50.000 vagas (Brasil, 2015). A participação de professores do município estudado foi pequena e não existiam profissionais matriculados nos últimos dois anos, sugerindo pouco interesse pelo assunto, além da falta de incentivo pela gestão pública.

Os docentes avaliados em nosso estudo, quando questionados sobre as dificuldades em implantar e manter a educação preventiva, reportaram à falta de profissionais capacitados em primeiro lugar e falta de conhecimento técnico em terceiro, indicando uma provável formação deficiente e a sua necessidade de orientação sobre como trabalhar com o tema. Um estudo norte-americano de programas de prevenção de base escolar (Caldwell, Bradley e Coffman, 2009) corrobora esse resultado, quando faz referência ao auxílio que os professores necessitam de profissionais da área técnica, para sua formação e na realização de atividades na escola. Apesar das suas dificuldades em lidar com o assunto, a importância do profissional da educação ficou demonstrada em estudo de revisão de programas de prevenção primária (Norberg, Kezelman e Lim, 2013), pois indicou que, quando o professor estava presente no processo, a propensão em produzir resultados estatisticamente significativos aumentava e quando se associava os docentes com profissionais da área técnica, os resultados eram ainda melhores.

A baixa participação da família apareceu na segunda posição, como uma dificuldade da educação preventiva, não se constituindo uma novidade, pois já foi identificada em representações sociais de professores no interior de Santa Catarina (Araldi et al, 2012), corroborando os resultados da Pesquisa Nacional de Saúde do Escolar (PeNSE 2012) (Horta et al, 2014), que verificou uma associação entre o uso de drogas ilícitas ao contato insuficiente entre a escola e os pais. A falta de políticas de incentivo à educação preventiva entrou em quarto lugar no relato dos professores, provavelmente sugerindo que as ações governamentais não estão chegando ao município de forma efetiva.

Outro dado que chamou atenção foi que os professores que possuíam atividades profissionais diferentes da educação sentiam-se mais preparados para realizar atividades 
preventivas $(41,4 \%)$ do que aqueles que trabalhavam somente com educação $(22,1 \%)$. Isso pode se relacionar com o fato de terem um horizonte mais amplo de experiências.

\section{Pontos fortes e limitações do estudo}

Como pontos fortes do estudo salienta-se que a pesquisa abrangeu todas as escolas públicas de ensino fundamental e médio do município, inclusive as rurais, de modo que foi possível entrevistar gestores e professores, com um índice de perdas de cerca de $9 \%$ no geral. Além disso, os instrumentos de pesquisa foram baseados em questionários já utilizados no censo escolar, não sendo uma novidade para os entrevistados. Como limitação, aponta-se o fato do estudo ter sido realizado em um município pequeno, com cerca de 40.000 habitantes, indicando que as extrapolações dos resultados devem ser realizadas com cuidado.

\section{CONCLUSÃO}

O estudo apontou que os profissionais da educação estudados carecem de orientação sobre como trabalhar com a educação preventiva. O fato de o tema ter estado presente no PPP em apenas pouco mais da metade das escolas, demonstra desconhecimento de um aspecto importante da Lei das Drogas e que poderia gerar um planejamento mais organizado sobre o assunto. O PSE que deveria subsidiar a direção das escolas com projetos sobre a educação preventiva, ainda precisa de maiores investimentos para chegar a todos os estabelecimentos e também requer maior conhecimento técnico sobre o tema, por parte dos seus profissionais.

A formação insuficiente dos professores, sobre o tema drogas, ficou evidenciada quando a grande maioria se declarou inapta a trabalhar com o assunto, apesar da quase totalidade acreditar que a escola deve desenvolver atividades preventivas.

A educação preventiva ao abuso de substâncias psicoativas pode apresentar resultados mais efetivos caso venha a ser realizada com abrangência maior, na linha da promoção da saúde e contemplando outros aspectos da vida, como o meio ambiente, a ética, a sexualidade e as diferenças culturais. Ficou evidenciado ainda que os gestores públicos deveriam proporcionar uma interação mais consistente entre o setor saúde e educação, colocando profissionais da área técnica para contribuir com a comunidade escolar nas suas ações preventivas e proporcionar uma formação mais adequada aos professores.

\section{0 que se sabe sobre o assunto}

Os professores já relataram em estudos qualitativos que não se sentiam capacitados em abordar a questão do uso de álcool e outras drogas.

A lei das drogas dispõe sobre a implantação de projetos pedagógicos de prevenção do uso indevido de drogas, nas instituições de ensino.

O Programa Saúde na Escola é o programa oficial que oferece suporte para as escolas quanto ao tema uso abusivo de álcool e outras drogas.

\section{O que o estudo apontou}

Dados quantitativos sobre a dificuldade que os professores relatam em abordar o tema (somente $24 \%$ se declararam aptos), corroborando os estudos qualitativos.

Apenas pouco mais da metade das escolas tem o tema no Projeto Político Pedagógico (57,5\%).

O Programa Saúde na Escola oferece atividades preventivas ao abuso de álcool e outras drogas em apenas $25 \%$ dos estabelecimentos que está presente. 


\section{REFERÊNCIAS}

Araldi, J.C; Njaine K; Oliveira, M.C; Ghizoni, A.C. (2012). Representações sociais de professores sobre o uso abusivo de álcool e outras drogas na adolescência: repercussões nas ações de prevenção na escola. Rev. Interface, Botucatu, 16(40), 135-46.

Barros, M.A; Pillon, S.C. (2007). Atitudes dos profissionais do Programa Saúde da Família diante do uso e abuso de drogas. Esc. Anna Nery Rev. Enferm. Rio de Janeiro, 1(4), 655 -62.

Brasil. (2006). Lei n.11343, de 23 de agosto de 2006. Institui o Sistema Nacional de Políticas Públicas sobre Drogas - SISNAD; prescreve medidas para prevenção do uso indevido, atenção e reinserção social de usuários e dependentes de drogas; estabelece normas para repressão à produção não autorizada e ao tráfico ilícito de drogas; define crimes e dá outras providências. Diário Oficial da União, Brasília, DF, 2006. Disponível em: <http://www.planalto.gov.br/ccivil_03/_ato2004-2006/2006/lei/l11343.htm>. Acesso em: 13 nov. 2014.

Brasil. (2007). Decreto n. 6.286, de 5 de dezembro de 2007. Institui o Programa Saúde na Escola PSE, e dá outras providências. Diário Oficial da União, Brasília, DF, 2007. Disponível em: $<$ http://portal.mec.gov.b r/index.php?option=com_content\&id= 14578 \%3aㅡ programasaude-nas-escolas\&Itemid=817>. Acesso em: 26 out. 2014.

Brasil. (2013). Ministério da Educação. INEP. Censo escolar 2013. Disponível em: <http://educacenso.inep.gov.br/escola/identificação>. Acesso em: 02 dez. 2013.

Brasil. (2013). Ministério da Educação. Parâmetros Curriculares Nacionais-Temas Transversais em Saúde. Disponível em: <http://portal.mec.gov.br/seb/arquivos /pdf/saude.pdf>. Acesso em: 01 set. 2013.

Brasil. (2015). Observatório Brasileiro de Informações sobre Drogas/ Secretaria Nacional de Políticas sobre Drogas/ Capacitação. Disponível em: <http://www.obid.senad.gov.br/portais/OBID/index.php>. Acesso em: 10 jan. 2015.

Caldwell, L.L; Bradley, S; Coffman, D. A. (2009). person-centered approach to individualizing a school-based universal preventive intervention. The American Journal of Drug and Alcohol Abuse, 35, 214-19.

Camarotti, A.C; Kornblit, A.L; Di Leo, P. F. (2013). Prevención del consumo problemático de drogas en la escuela: estrategia de formación docente en Argentina utilizando TIC. Rev. Interface, Botucatu, 17(46), 695-703.

Degenhardt, L. et al. Global burden of disease attributable to illicit drug use and dependence: findings from the Global Burden of Disease Study 2010. Lancet, 382, 1564-74.

Horta, R. L. et al. (2014). Uso na vida de substâncias ilícitas e fatores associados entre escolares brasileiros, Pesquisa Nacional de Saúde do Escolar (PeNSE 2012). Rev. Bras. Epidem. Suppl. PeNSE, 31-45. 
Knevitz, M. F; Béria, J. U; Schermann, L. B. (2017). Percepções e demandas de professores sobre educação preventiva ao abuso de álcool e outras drogas. Rev. Holos, 33(4), 357-370.

Kornblit, A. L. (2010). La promoción de La salud entre los jóvenes. Acta Psiquiat. Psicol. Am. Lat., 56(3), 217-26.

Midford, R. et al. (2007). Is Australia 'fair dinkum' about drug education in schools? Drug and Alcohol Review, 26(4), 421-27.

Midford, R. et al. (2012). Drug education in victorian schools (DEVS): the study protocol for a harm reduction focused school drug education trial. BMC Public Health, 12, 112-19.

Norberg, M. M; Kezelman, S; Lim-Howe, N. (2013). Primary prevention of cannabis use: A systematic review of randomized controlled trials. Rev. Plos One, 8(1).

Santos, E. O; Oliveira, M. F. S. S; Kauark, F. S; Manhães, F. C. (2011). Abordagem sobre a prevenção de drogas no contexto escolar. Rev. Cient. Internacional, 4(17), 18-40.

Sodelli, M. (2010). A abordagem proibicionista em desconstrução: compreensão fenomenológica existencial do uso de drogas. Rev. Ciência Saúde Coletiva, 15(3), 637-44.

Stephens, P. C. et al. (2009). Universal school-based substance abuse prevention programs: Modeling targeted mediators and outcomes for adolescent cigarette, alcohol and marijuana use. Drug and Alcohol Dependence, 102, 19-29.

Tupper, K. W. (2008). Teaching teachers to just say "know": Reflections on drug education. Teaching and Teacher Education, 24, 356-67.

United Nations Office on Drugs and Crime (UNODC). (2014). World Drug Report: Executive Summary. Viena, jun. 2014. Disponível em: https://www.unodc.org/doc/wdr 2016/world_drug_report_2016_web.pdf. Acesso em: 10 jul. 2016. 